\title{
The efficacy of enhanced recovery after surgery for elderly patients receiving surgery for intertrochanteric fracture: a study protocol for a randomized blinded controlled clinical trial
}

Mengchen Yin ( $\nabla$ yinmengchen0513@126.com )

Longhua Hospital https://orcid.org/0000-0001-7404-1495

Yinjie Yan

Longhua Hospital

Zhaoxiang Fan

Longhua Hospital

Niankang Fang

Longhua Hospital

Hongbo Wan

Longhua Hospital

\section{Wen Mo}

Longhua Hospital

\section{Xuequn Wu}

Longhua Hospital

Study protocol

Keywords: intertrochanteric fracture, enhanced recovery after surgery, perioperative period, integrating TCM with and western medicine, randomized controlled trial

Posted Date: January 8th, 2020

DOI: https://doi.org/10.21203/rs.2.20404/v1

License: (c) (1) This work is licensed under a Creative Commons Attribution 4.0 International License. Read Full License 


\section{Abstract}

Background: Intertrochanteric fracture (ITF) is increasing with the rapid increase in the aging population, often cause a high mortality rate in old patients and increase the economic burden of the family and society. ERAS is a powerful guarantee for patients to accelerate their recovery after surgery. TCM promote repair of injured tissues and eliminate traumatic aseptic inflammation. Therefore, this prospective randomized controlled clinical trial aims to evaluate the clinical effect of the evidence-based ERAS pathway of integrating TCM with and western medicine on perioperative outcomes in ITF patients undergoing intramedullary fixation, and provide reliable evidence-based data for applying the program to clinical practice.

Methods/design: We will conduct a prospective randomized, blinded, controlled trial to compare the effectiveness of ERAS care pathway with traditional care pathway, and to investigate whether the ERAS care pathway can improve the perioperative outcome in ITF patients undergoing intramedullary fixation. A total of 60 patients with ITF will be enrolled and treated with the two care pathway, respectively. Length of stay, economic indicators, Harris score, VAS score, time get out of bed, 30-day readmission rates, postoperative transfusion rates, discharge to home and mortality will be evaluated. Any signs of acute adverse reactions will be recorded at each visit during treatment.

Discussion: Although an evidence-based process using the best available literature and Delphi expertopinion method has been used to establish an ERAS pathway of integrating TCM with western medicine. But, there is a lack of consensus about its effectiveness. This trial will provide convincing evidence about the effect of ERAS pathway

Trial registration: Registered on 12 Oct 2019; Trial number is ChiCTR190t0026487

Keywords: intertrochanteric fracture; enhanced recovery after surgery; perioperative period; integrating TCM with and western medicine; randomized controlled trial

\section{Introduction}

With the aging of society, the incidence of intertrochanteric fracture is increasing. It is easy to bring bedsore, urinary tract infection, lung infection and other complications. Intertrochanteric fracture has become a major public health issue with a high mortality rate for the old patients and increase the economic burden. ${ }^{1,2}$ Currently, intertrochanteric fracture is treated with both surgical and non-surgical methods. Surgical treatment has great advantages in alleviating pain, restoring hip function, improving quality of life and avoiding the complications such as deep vein thrombosis and cardiovascular accident caused by long-term bed rest, so surgical treatment is the first choice of treatment. ${ }^{3-6}$ Currently PFNA (proximal femoral nail anti-rotation, PFNA) is a mature surgical treatment for senile intertrochanteric fractures, which is characterized by maintaining strong fixation, biomechanical stability and minimally invasion. ${ }^{7,8}$ 
ERAS (Enhanced Recovery after Surgery) is one of the two important development directions leading the development of modern surgery in the 21 st century, which is first proposed by Kehlet in $1997 .{ }^{9}$ ERAS aims to integrate the perioperative diagnosis and treatment concept by optimizing a series of effective approaches and methods with evidence-based medical evidence, so as to reduce trauma invasion and alleviate the stress reaction caused by surgery. The core of the ERAS is a powerful guarantee for patients

to accelerate their recovery after surgery. ${ }^{10-13}$ At present, ERAS has been applied in the treatment of many orthopedic diseases including artificial joint replacement. ${ }^{14}$

Currently, major efforts are being conducted to transfer the lessons learned from other surgical specialties and incorporate ERAS protocols into postoperative care of patients undergoing hip surgery. ERAS protocols for artificial joint replacement have been described that it can reduce postoperative mortality and increased satisfaction for patients. However, to my best knowledge, ERAS programs for patients with intertrochanteric fracture hasn't been described. Furthermore, traditional Chinese medicine including acupuncture, manipulative therapy and oral Chinese medicine can improve blood circulation in local tissues, relax tense and spasmodic muscles, promote repair of injured tissues and eliminate traumatic aseptic inflammation. Therefore, it is of great significance to establish an effective plan and management pathway integrated Chinese and western medicine to accelerate postoperative recovery of intertrochanteric fractures. With the objective in mind, we have conducted a systematic literature search to guarantee the comprehensiveness of the study, Delphi expert-opinion method was used to establish the ERAS pathway. Here, ERAS programs consist of a multidisciplinary evidence-based approach to preoperative management, intraoperative control and rehabilitation for intertrochanteric fracture and preoperative multidisciplinary collaboration had been already established.

Therefore, this prospective, randomized, controlled clinical trial aims to evaluate the clinical effect of an evidence-based ERAS pathway integrated Chinese and western medicine on perioperative outcomes in intertrochanteric fracture patients undergoing intramedullary fixation, and provide reliable evidence-based data

\section{Materials And Methods Study design}

This study is a prospective, outcome assessor- and data analyst-blinded, randomized controlled design study that compared an ERAS care pathway cohort with standardized care pathway cohort. Emergency admissions with a primary diagnosis of intertrochanteric fracture between January 2020 and July 2021 in our hospital will be selectively enrolled. All patients received their operation at our hospital where elective surgeries take place predominantly, and they will be treated with PFNA intramedullary fixation at our institution.

The objective of this proposed study is to investigate whether ERAS care pathway could lead to perioperative improvement in intertrochanteric fracture patients undergoing intramedullary fixation. The 
principal investigator $(\mathrm{PI})$ is responsible for the overall project and organizing Steering Committee meetings. Pls of sub-center departments are responsible for gathering experts to carry out the project. An independent Steering Committee will be responsible for affairs such as participants' safety, meetings, recruitment and follow-up of participants, and quality control. The coordinating center is responsible for communicating protocol modifications and providing materials. This trial includes a 2-week treatment period and a 3-month follow-up period. Outcome assessments will be conducted at baseline, as well as at $3,14,30$ and 90 days. (Fig. 1)

\section{Eligibility criteria}

\section{Inclusion criteria}

Participants who meet the criteria below are eligible: ${ }^{15}$

- The patients are over 75 years old.

- X-ray or CT indicate unstable intertrochanteric fractures.

- Treated with PFNA intramedullary fixation

- Being willing to undergo surgery with the ERAS pathway.

- Being willing to give informed consent

\section{Exclusion criteria}

The exclusion criteria are as follows: ${ }^{15}$

- Patients are with open fractures or pathological fracture caused by tumor, infection or tuberculosis.

- Patients are with congenital hip dysplasia or osteonecrosis of the femoral head.

- Hip surgery before enrollment.

- Failure to understand or sign informed consent.

\section{Patient population and recruitment procedure}

Participants will be recruited from Longhua Hospital affiliated with the Shanghai University of Traditional Chinese Medicine. Prospective participants will be interviewed by the coordinators and informed of the eligibility criteria and the procedure. Those who are eligible and willing to participate the study will be screened initially by baseline assessment and then diagnosed based upon clinical manifestations, physical examination and imaging. Participants will be informed that participating the trial was absolutely voluntary and withdrawal from the trial can be made at any time. In case of withdrawal, the data collected will not be deleted and will be used in the final analyses. A data compilation form including all variables of interest and all potential risks will be completed by the corresponding research center. The information obtained will be stored in an electronic database for subsequent statistical analysis. 
Recruitment will start in January 2020, and is expected to end in July 2021. The final follow up of all participants will be completed on 30 Sept 2021. The overview of the participant processing and the schedule of evaluation is provided in Fig. 1.

\section{Patient and public involvement}

Patients and/or public were not involved in the study design and study enrolment

\section{Ethics}

This study is to be conducted in accordance with the principles of the Declaration of Helsinki and has been approved by Sichuan Regional Ethics Review Committee on Traditional Chinese Medicine (TCM). ${ }^{16}$ The trials have been approved by the appropriate Institutional Review Boards. The clinical trial has been approved by the Institutional Review Board of Longhua Hospital, Shanghai University of TCM. All participants will be given sufficient time to reach a decision to sign the consent form prior to the study in compliance with Good Clinical Practice Guidelines that guides the appropriate use of TCM in clinical practice. Then the participants will be randomized into two groups of different treatments. Reporting will be guided by the CONSORT statement. ${ }^{17,18}$

\section{Intervention}

\section{ERAS care pathway}

An evidence-based process using the best available literature and Delphi expert-opinion method was used to establish the ERAS pathway integrated Chinese and western medicine. The basic components of the multi-disciplinary ERAS pathway which we used are shown in Table 1. The principles include educational program, management of nutrition, management of dietary, management of sleep, management of pain selection of anesthesia, control of bleeding, management of body temperature, prevention of infection, management of anesthesia, management of rehydration management of drainage tube, control of nausea and vomiting, management of activity.

\section{Traditional care pathway}

There was no detailed plan in the controlled group, anti-infection, anti-coagulation and other measures were taken to prevent complications during the perioperative period.

\section{Randomization and Allocation}


After the screening, patients will be randomized into two groups with an allocation ratio of 1:1. The randomization will be generated via SAS PROC PLAN software (SAS Inc., Cary, NC, USA) by an independent 3rd-party clinical research organization (Institute of Basic Research in Clinical Medicine, China Academy of Chinese Medical Science) and concealed from the researchers by a senior data manager who is not involved in the study. The group assignment list will be sealed in opaque envelopes and be opened by the researchers following informed consent procedures and baseline testing.

\section{Blinding}

All the investigators, physicians, nurses, assessors, analysts, and participants will be blinded to the group assignment until the end of the trial, when all statistical analyses are finished. If, after the first administration, any adverse event potentially related to the treatment occurs, the study physician will reevaluate the participant, and PI will decide whether the non-blinded procedure is necessary. If nonblinding is required, the allocation information will be provided.

\section{Outcome measurements}

\section{Primary outcome measurements}

LOS (length of stay) associated with intertrochanteric fracture is a major public health issue due to the aging population and it is the most objective outcome of evaluating the recovery pathway. Furthermore, high LOS would correspond with increases in postoperative complications. ${ }^{14,19}$ So we chooses the LOS at discharge as the primary outcome measurement to assess the speed of recovery. Economic indicators including major medical expenses, bed expenses, drug expenses, inspection expenses, operation expenses, cost of anesthesia, nursing care and blood transfusion are the other primary outcome measurement.

\section{Secondary outcome measurements}

Postoperative joint specific function will be measured using the Harris score, which is widely used to assess the joint function of life for intertrochanteric fractures patients. The Harris score is composed of 10 questions, 2 questions (ROM and absence of deformity) for the physician physical examination component and 8 questions for the patient-reported outcome component. ${ }^{20-24}$ VAS is a reliable and valid measurement of pain. It has a horizontal, 100-mm-long line, with "no pain" recorded on the left side (score: 0 ) and "pain as bad as it could be" on the right side (score: 10$) \cdot{ }^{25}$ The other secondary outcomes included time to get out of bed, 30 day readmission rates, postoperative transfusion rates, discharge to home and mortality. 


\section{Safety Assessments}

Infection, deep venous thrombosis, cardiovascular accidents will be recorded at each visit during treatment.

\section{Sample size calculation}

We calculate the sample size according to our primary study. We conducted a preliminary clinical trial and pilot trial about ERAS care pathway versus traditional care pathway from January 2018 to May 2018. The primary outcome parameter was the LOS. Based on the previous results, we found that primary outcome parameter of ERAS care pathway group was 5.8 days, and that of the traditional care pathway group was 9.2 days. According to the formula of the rate in completely random design,

$$
\mathrm{n}_{1}=\mathrm{n}_{2}=\frac{\left[u_{\alpha / 2} \sqrt{2 \bar{p}(1-\bar{p})}+u_{\beta} \sqrt{p_{1}\left(1-p_{1}\right)+p_{2}\left(1-p_{2}\right)}\right]}{\left(p_{1} \cdot p_{2}\right)^{2}}
$$

among which, $n_{1}$ and $n_{2}$ were the number of patients in the two respectively, $u_{a / 2}=1.96$ when type 1 error is $0.05, u_{\beta}=1.282$ when type II error is 0.1 in two-sided tests. $\bar{p}$ is the mean of $p_{1}$ and $p_{2} \cdot{ }^{26} A$ two-sided $5 \%$ significance level and $90 \%$ power in detecting treatment differences were considered, and the above relevant data were input into SPSS 20.0 software. This number of patients actually provided less than $80 \%$ power, considering an estimated dropout rate of $20 \%$. Therefore, we will recruit a total of 72 patients with 36 patients in each group.

\section{Statistical analyses}

Prior to all analyses, a detailed statistical analysis protocol will be developed. All data will be analyzed in the clinical research center of Longhua Hospital affiliated to Shanghai University of TCM by statisticians blinded to allocation using the SPSS 20.0 statistical software (SPSS Inc., Chicago, Illinois). Efficacy and safety analyses will be conducted according to the intention-to-treat principle using the "last observation carried forward" rule. Before randomization, baseline characteristics will be collected as descriptive statistics for each patient, including gender, age, BMI, duration of symptoms, preoperative red blood cell count and preoperative hemoglobin count. The data analysis of the primary outcome is based on the perprotocol population as a supportive analysis. Mean, standard deviation, median, quartiles and inter quartiles for continuous variables, and frequency for categorical variables will be calculated. Continuous variable followed the normal distribution will be presented as means with standard deviations (SDs) and calculated by an independent sample Student's t-test, otherwise the data will be expressed as medians with ranges, and non-parametric tests will be used. Categorical variables will be expressed as number (\%) and analyzed by $\chi^{2}$ test or Fisher's exact test. A P value of less than 0.05 is defined as statistical 
significant with 2-sided $90 \%$ confidence intervals (Cls). Missing data will be input with the last observed response carried forward for all measures using the "last-value-carried-forward" principle.

\section{Quality control}

Prior to the clinical trial, we will carry out unified training to make sure all the physicians, nurses, and assessors involved fully understand the process of the trial, including selecting patient screening and selection, case report form writing and manipulation details. To guarantee the quality of the whole trial, rigorous monitoring will be performed by 3 trained quality supervisors. During the trial, supervisors will check on case report forms and intervention twice a month. After verifying the case report forms, data will be input into the database by two full-time research members independently. The standard operating procedures (SOP) will be invariably followed. Drop-outs, withdrawals (and the reasons) and any compliance of all patients occurring will be recorded in detail by the inspectors throughout the treatment and follow-up period.

\section{Discussion}

The surgical stress response associated with major surgery describes fundamental metabolic changes that lead to increased catabolism, immunosuppression, free radical production, and hypercoagulable states. These physiologic alterations have been linked to changes in organ function resulting in undesirable postoperative morbidity, complications, pain, fatigue and extended convalescence. ERAS attempts to decrease the surgical stress response to minimize postoperative complications and improve surgical outcomes and functional rehabilitation after major surgery.

Intertrochanteric fractures commonly occurred in the elderly and caused high mortality rate due to the complications of loss of walking ability. Due to the great importance of good functional outcome and avoidance of serious postoperative complications, the general consensus is that early surgery is the first choice of treatment for the elderly patients and PFNA is a mature surgical procedure.

Several clinical literatures describing ERAS on arthroplasty have been published previously in the literature, but there is a lack of prospective high-quality data from larger cohorts on it for improved recovery after surgery. In particular, we are unaware of published ERAS program on the field of intertrochanteric fracture. Based on the theory of TCM, manipulations, acupuncture and oral Chinese medicine are of great significance in promoting fracture healing and improving patients' whole body state. With the objective, we have conducted a systematic literature search including Wan Fang Data, CNKI databases, Vip Journal Integration Platform, Chinese BioMedical databases, PubMed, MEDLINE, EMBASE, Cochrane Library and ISI web of knowledge, ERAS pathway integrated Chinese and western medicine was established by Delphi expert-opinion process including ten experts. The new ERAS project is consist of a multidisciplinary evidence-based approach to preoperative management, intraoperative control and rehabilitation. 
To our best knowledge, our study is the first elaborately designed, randomized, controlled trial to evaluate the clinical effect of the ERAS project integrated Chinese and western medicine on perioperative outcomes in intertrochanteric fracture patients undergoing PFNA. A hallmark of ERAS is coordination between care services before and after surgery and continual evaluation of postoperative course with attention toward pain control, functional recovery, and patient satisfaction to improve standards of care. So the study is designed as a comprehensive study of LOS, economic indicators, pain relief, functional outcome and complications. Outcome measurements are widely used in research to establish baselines, evaluate the effect of an intervention, and motivate patients' self-evaluation. LOS was the most commonly assessed metric in comparative analyses. And a comparative reduction in LOS was reported in most studies using the ERAS protocol. Improved cost-effectiveness of surgical interventions is also primary objective when implementing strategies for faster recovery in this study. The calculation of costeffectiveness provided is based on data provided by our Department of Finance. The study is also to evaluate whether ERAS program can led to a reduction in hospitalization costs, care costs and nursing costs, which supporting the cost-effectiveness of "fast-track" protocols in PFNA surgery. The VAS measurements, which have been found valid, reliable and easy to apply in researches, are often used as the criterion standard to evaluate the pain intensity. The Harris score, a self-administered questionnaire, has been widely adopted as the criterion standard to estimate disease activity for its reliability and validity. Another aim of study lies in reducing both intra- and postoperative adverse events and complication, which have the potential to impair the patients' perioperative well-being and to prolong recovery. Especially in elderly patients, or in those with severe comorbidities, strict patient selection is key to both eligibility for safe ERAS and to treatment in specialized short-stay clinics that may not have an ICU available. Administration of prophylactic medication against infections and thrombosis, prevention of hypothermia and fluid imbalance, as well as operative measures have therefore become integral to successful implementation of ERAS. ${ }^{27-30}$

The lack of good quality RCTs in the field leaves us with notable gaps in our knowledge, and in clinical practice many decisions have to be taken without the benefit of high-quality evidence. So we decide to conduct a prospective, randomized, controlled clinical trial, which also ensures the compliance of participants and meets ethical considerations, to closely detect the clinical efficacy of ERAS program. The present study is built on our preliminary open experiment: a small-sample-sized, randomized and controlled trial of ERAS program for patients with intertrochanteric fracture. The result of the preliminary trial has showed that LOS of ERAS care pathway group was 5.8 days, and that of the traditional care pathway group was 9.2 days.

We hope that this trial, with a larger number of patients, can provide adequate statistical power to further analyze and explore the efficacy of ERAS. Thus, quality control is vital to the whole study, as we described in the protocol. To perform a reliable study, we will carry out unified training to make sure all the physicians, nurses, and assessors involved in the trials fully understand the process and details of ERAS program before the clinical trial. Hopefully, this trial will produce high-quality evidence pertaining to the efficacy and safety of the ERAS in treating patients with intertrochanteric fracture. The results will aid in 
clinical decision-making for the management and provide useful information that can be incorporated into future guidelines.

\section{Abbreviations}

\section{ERAS}

Enhanced Recovery after Surgery

LOS

length of stay

PFNA

proximal femoral nail anti-rotation

\section{Declarations}

\section{Ethics approval and consent to participate}

This study is to be conducted in accordance with the principles of the Declaration of Helsinki and has been approved by Sichuan Regional Ethics Review Committee on Traditional Chinese Medicine.16 Details of the trial program have been approved by the appropriate Institutional Review Boards

\section{Consent for publication}

Not applicable

\section{Availability of data and materials}

The datasets used and/or analyzed during the current study are available from the corresponding author on reasonable request. Ethics approval and consent to participate. The study design, procedures, and informed consent procedure were approved by the Longhua Hospital. Consent to participate will be obtained from the participants.

\section{Competing interests}

The authors declare that they have no competing interests

\section{Funding}

This work was sponsored by research grants from

\section{Authors' contributions}

YMC, YYJ and FZX are co-first authors of this manuscript, contributing equally to the design, conduct of the trials and drafting the manuscript. All authors participated in the design of the study and performed the trial. MW and WXQ are co-corresponding author of this manuscript, contributing equally supervised and coordinated the clinical trial. All authors read and approved the final manuscript. 


\section{Acknowledgements}

We thank all the medical staff in the department of orthopedics and traumatology of Longhua Hospital affiliated to Shanghai University of Traditional Chinese Medicine for their meticulous care of patients who have given us great help. We also thank the patients for their support and trust, and their medical treatment is also a great help to us.

\section{Author details}

1: Department of Orthopaedics, Longhua Hospital, Shanghai University of Traditional Chinese Medicine, No.725 South Wanping Road, Shanghai 200032, China.

\section{References}

1. Bhowmick K, Matthai T, Boopalan PRJ, Jepegnanam TS. Decision making in the management of malunion and nonunion of intertrochanteric fractures of the hip. Hip Int. Jul 15 2019:1120700019863410.

2. Cho SH. Outcomes of dynamic hip screw augmented with trochanteric wiring for treatment of unstable type A2 intertrochanteric femur fractures. Injury. Mar 2019;50(3):816.

3. Jung EY, Oh IT, Shim SY, Yoon BH, Sung YB. The Effect of Valgus Reduction on the Position of the Blade of the Proximal Femoral Nail Antirotation in Intertrochanteric Hip Fractures. Clin Orthop Surg. Mar 2019;11(1):36-42.

4. Imerci A, Aydogan NH, Tosun K. The effect on outcomes of the application of circumferential cerclage cable following intramedullary nailing in reverse intertrochanteric femoral fractures. Eur $J$ Orthop Surg Traumatol. May 2019;29(4):835-842.

5. Han YH, Jeong HJ, Sohn MH, Yoon SJ, Lim ST. Incidence and severity of femoral head avascularity after femoral neck or intertrochanteric fractures on preoperative bone single photon emission computed tomography/computed tomography: preliminary study. Nucl Med Commun. Mar 2019;40(3):199-205.

6. Li J, Zhang $\mathrm{L}$, Zhang $\mathrm{H}$, et al. Effect of reduction quality on post-operative outcomes in 31-A2 intertrochanteric fractures following intramedullary fixation: a retrospective study based on computerised tomography findings. Int Orthop. Aug 2019;43(8):1951-1959.

7. Makki D, Matar HE, Jacob N, Lipscombe S, Gudena R. Comparison of the reconstruction trochanteric antigrade nail (TAN) with the proximal femoral nail antirotation (PFNA) in the management of reverse oblique intertrochanteric hip fractures. Injury. Dec 2015;46(12):2389-2393.

8. Nishiura T, Nozawa M, Morio $\mathrm{H}$. The new technique of precise insertion of lag screw in an operative treatment of trochanteric femoral fractures with a short intramedullary nail. Injury. Oct 2009;40(10):1077-1083.

9. Kehlet $\mathrm{H}$. Multimodal approach to control postoperative pathophysiology and rehabilitation. $\mathrm{Br} \mathrm{J}$ Anaesth. May 1997;78(5):606-617. 
10. Ljungqvist O, Scott M, Fearon KC. Enhanced Recovery After Surgery: A Review. JAMA Surg. Mar 1 2017;152(3):292-298.

11. Grant MC, Yang D, Wu CL, Makary MA, Wick EC. Impact of Enhanced Recovery After Surgery and Fast Track Surgery Pathways on Healthcare-associated Infections: Results From a Systematic Review and Meta-analysis. Ann Surg. Jan 2017;265(1):68-79.

12. Malczak P, Pisarska M, Piotr M, Wysocki M, Budzynski A, Pedziwiatr M. Enhanced Recovery after Bariatric Surgery: Systematic Review and Meta-Analysis. Obes Surg. Jan 2017;27(1):226-235.

13. Pearson KL, Hall NJ. What is the role of enhanced recovery after surgery in children? A scoping review. Pediatr Surg Int. Jan 2017;33(1):43-51.

14. Zhu S, Qian W, Jiang C, Ye C, Chen X. Enhanced recovery after surgery for hip and knee arthroplasty: a systematic review and meta-analysis. Postgrad Med J. Dec 2017;93(1106):736-742.

15. Kang $\mathrm{Y}$, Liu J, Chen $\mathrm{H}$, et al. Enhanced recovery after surgery (ERAS) in elective intertrochanteric fracture patients result in reduced length of hospital stay (LOS) without compromising functional outcome. J Orthop Surg Res. Jul 9 2019;14(1):209.

16. Fuson RL, Sherman M, Van Vleet J, Wendt T. The conduct of orthopaedic clinical trials. J Bone Joint Surg Am. Jul 1997;79(7):1089-1098.

17. Begg $C$, Cho $M$, Eastwood $S$, et al. Improving the quality of reporting of randomized controlled trials. The CONSORT statement. JAMA. Aug 28 1996;276(8):637-639.

18. Schulz KF, Altman DG, Moher D. CONSORT 2010 statement: updated guidelines for reporting parallel group randomized trials. Ann Intern Med. Jun 1 2010;152(11):726-732.

19. Dietz N, Sharma M, Adams S, et al. Enhanced Recovery After Surgery (ERAS) for Spine Surgery: A Systematic Review. World Neurosurg. Jul 2 2019;130:415-426.

20. Bagheri Z, Jafari P, Faghih M, Allahyari E, Dehesh T. Testing measurement equivalence of the SF-36 questionnaire across patients on hemodialysis and healthy people. Int Urol Nephrol. Dec 2015;47(12):2013-2021.

21. Jenkinson C, Peto V, Coulter A. Making sense of ambiguity: evaluation in internal reliability and face validity of the SF 36 questionnaire in women presenting with menorrhagia. Qual Health Care. Mar 1996;5(1):9-12.

22. Kili S, Wright I, Jones RS. Change in Harris hip score in patients on the waiting list for total hip replacement. Ann R Coll Surg Engl. Jul 2003;85(4):269-271.

23. Mahomed NN, Arndt DC, McGrory BJ, Harris WH. The Harris hip score: comparison of patient selfreport with surgeon assessment. J Arthroplasty. Aug 2001;16(5):575-580.

24. Soderman $\mathrm{P}$, Malchau $\mathrm{H}$. Is the Harris hip score system useful to study the outcome of total hip replacement? Clin Orthop Relat Res. Mar 2001(384):189-197.

25. Campbell WI, Lewis S. Visual analogue measurement of pain. Ulster Med J. Oct 1990;59(2):149-154.

26. Fayers P. Approaches to sample size estimation in the design of clinical trials--a review. By A. Donner, Statistics in Medicine, 3, 199-214 (1984). Stat Med. Sep 15 1993;12(17):1643. 
27. Hoeksma HL, Van Den Ende CH, Ronday HK, Heering A, Breedveld FC. Comparison of the responsiveness of the Harris Hip Score with generic measures for hip function in osteoarthritis of the hip. Ann Rheum Dis. Oct 2003;62(10):935-938.

28. Hung M, Hon SD, Cheng $C$, et al. Psychometric Evaluation of the Lower Extremity Computerized Adaptive Test, the Modified Harris Hip Score, and the Hip Outcome Score. Orthop J Sports Med. Dec 2014;2(12):2325967114562191.

29. Kalairajah Y, Azurza K, Hulme C, Molloy S, Drabu KJ. Health outcome measures in the evaluation of total hip arthroplasties-a comparison between the Harris hip score and the Oxford hip score. $J$ Arthroplasty. Dec 2005;20(8):1037-1041.

30. Li Z, Wang Q, Li B, Bai B, Zhao Q. Influence of enhanced recovery after surgery programs on laparoscopy-assisted gastrectomy for gastric cancer: a systematic review and meta-analysis of randomized control trials. World J Surg Oncol. Nov 23 2017;15(1):207.

\section{Table}

Table 1. The basic components of the multi-disciplinary ERAS pathway which we used 


\begin{tabular}{|c|c|c|}
\hline \multirow[t]{16}{*}{ Preoperative } & \multirow{5}{*}{$\begin{array}{l}\text { Educational } \\
\text { program }\end{array}$} & (1) understand the patient, assess the condition \\
\hline & & (2) psychological, nutrition, surgery, rehabilitation education \\
\hline & & (3) good communication \\
\hline & & (4) emphasize active function exercise \\
\hline & & $\begin{array}{l}\text { (5) advocate deep breathing, upper limbs pull rings and other } \\
\text { cardiopulmonary exercise }\end{array}$ \\
\hline & \multirow[t]{4}{*}{$\begin{array}{l}\text { Management } \\
\text { of nutrition }\end{array}$} & $\begin{array}{l}\text { (1) if there is hypoalbuminemia and severe anemia, actively look for } \\
\text { the original disease and correct it }\end{array}$ \\
\hline & & (2) when necessary, human serum albumin $10 \mathrm{~g} \mathrm{lvgtt}$ \\
\hline & & $\begin{array}{l}\text { (3) megaloblyte anemia : folate 5-10mg Po Tid+ vitamin B12 0.5mg } \\
\text { Im Tiw }\end{array}$ \\
\hline & & $\begin{array}{l}\text { (4) iron deficiency anemia: EPO 10,000 IU Ih Tiw+ Ferrous succinate } \\
0.2 \text { g Po Tid }\end{array}$ \\
\hline & \multirow{5}{*}{$\begin{array}{l}\text { Management } \\
\text { of dietary }\end{array}$} & (1) eat a high protein diet \\
\hline & & (2) before anesthesia $6 \mathrm{~h}$ fast protein liquid (such as milk, broth) \\
\hline & & $\begin{array}{l}\text { (3) before anesthesia } 4 \mathrm{~h} \text { fast carbohydrates (such as rice porridge, } \\
\text { steamed bread) }\end{array}$ \\
\hline & & (4) $2 \mathrm{~h}$ before anesthesia, do not drink clear liquid \\
\hline & & $\begin{array}{l}\text { (5) when necessary, } 250-500 \mathrm{ml} \text { glucose was dropped } 2-3 \mathrm{~h} \text { before } \\
\text { operation }\end{array}$ \\
\hline & $\begin{array}{l}\text { Management } \\
\text { of sleep }\end{array}$ & (1) Sedative hypnotic or anti-anxiety drugs \\
\hline & $\begin{array}{l}\text { Management } \\
\text { of pain }\end{array}$ & $\begin{array}{l}\text { (1) Routine use of anti-inflammatory analgesics such as celecoxib } \\
200 \mathrm{mg} \text { Po Bid }\end{array}$ \\
\hline \multirow[t]{6}{*}{ Intraoperative } & \multirow{2}{*}{$\begin{array}{l}\text { Selection of } \\
\text { anesthesia }\end{array}$} & (1) general anesthesia (laryngeal mask or endotracheal intubation) \\
\hline & & $\begin{array}{l}\text { (2) combined with local infiltration anesthesia: ropivacaine } 200 \\
\text { mg+80 } \mathrm{ml} \text { saline was injected into the incision and surrounding } \\
\text { deep needle }\end{array}$ \\
\hline & \multirow[t]{2}{*}{$\begin{array}{l}\text { Control of } \\
\text { bleeding }\end{array}$} & $\begin{array}{l}\text { (1) blood pressure control: systolic blood pressure control in 90- } \\
110 \mathrm{mmhg}\end{array}$ \\
\hline & & $\begin{array}{l}\text { (2) bleeding control: } 5-10 \mathrm{~min} \text { before skin incision, tranexamic acid } \\
\text { should be dropped } 15-20 \mathrm{mg} / \mathrm{kg}\end{array}$ \\
\hline & \multirow{2}{*}{$\begin{array}{l}\text { Management } \\
\text { of body } \\
\text { temperature }\end{array}$} & $\begin{array}{l}\text { (1) monitor and dynamically adjust the operating room } \\
\text { temperature, do a good job of keeping warm }\end{array}$ \\
\hline & & $\begin{array}{l}\text { (2) reduce limb exposure, for patients covered inflatable heating } \\
\text { blanket }\end{array}$ \\
\hline
\end{tabular}


(3) the infusion of liquid will be first heated to $37^{\circ} \mathrm{C}$

Prevention of infection

(1) ensure the operating room environment, control the number of patients involved in the operation

(2) strict disinfection towel, as far as possible to shorten the operation time and reduce the surgical trauma, the operation field repeatedly rinse

(3) preoperative $0.5-2 \mathrm{~h}$ intravenous antibiotics

(4) if the operation time exceeds $3 \mathrm{~h}$, or blood loss $>1500 \mathrm{ml}$ with the second dose

(5) the effective coverage time of antibacterial drugs includes the whole surgical process and 4 hours after surgery, and the total prevention time is no more than $24 \mathrm{~h}$

Postoperative Management

anesthesia
(1) general anesthesia wake up: drink water before eating

(2) moxapride 5mg Po Tid to improve gastrointestinal motility

(3) Selection of anesthesia

Management

of rehydration
(1) avoid a large amount of fluid replacement: infusion volume from 25 to $40 \mathrm{ml}(\mathrm{Kg} / \mathrm{d})$ is appropriate

(2) control the infusion speed: the infusion speed of elderly patients is from 100 to $120 \mathrm{ml} / \mathrm{h}$ is appropriate

(3) monitor blood routine, liver function, kidney function and cardiac function indicators
Management of drainage tube

(1)No drainage or catheter was placed

$\begin{aligned} & \text { Control of } \\ & \text { nausea and } \\ & \text { vomiting }\end{aligned}$
$\begin{aligned} & \text { Management } \\ & \text { of sleep }\end{aligned}$
$\begin{aligned} & \text { Management } \\ & \text { of pain }\end{aligned}$

(1) Intraoperative intravenous use of dexamethasone $10 \mathrm{mg}$

(2) use ondansetron when necessary

(1) Sedative hypnotic or anti-anxiety drugs

(1) use of automatic analgesia pump for three days

(2) sequential use of anti-inflammatory and analgesic drugs, such as celecoxib $200 \mathrm{mg}$ Po Bid (recommended reduction of $50 \%$ for liver damage and elderly patients)

Management of activity

(1) emphasis on early hip, knee and ankle active flexion and extension function exercise, to increase muscle strength

(2) exercise passive joint flexion and extension of hip, knee and ankle joints with the help of the physician and CPM, at least 3 times a day, at least 15 minutes each time

(3) asked frequently turn over, clap back 


\section{Figures}

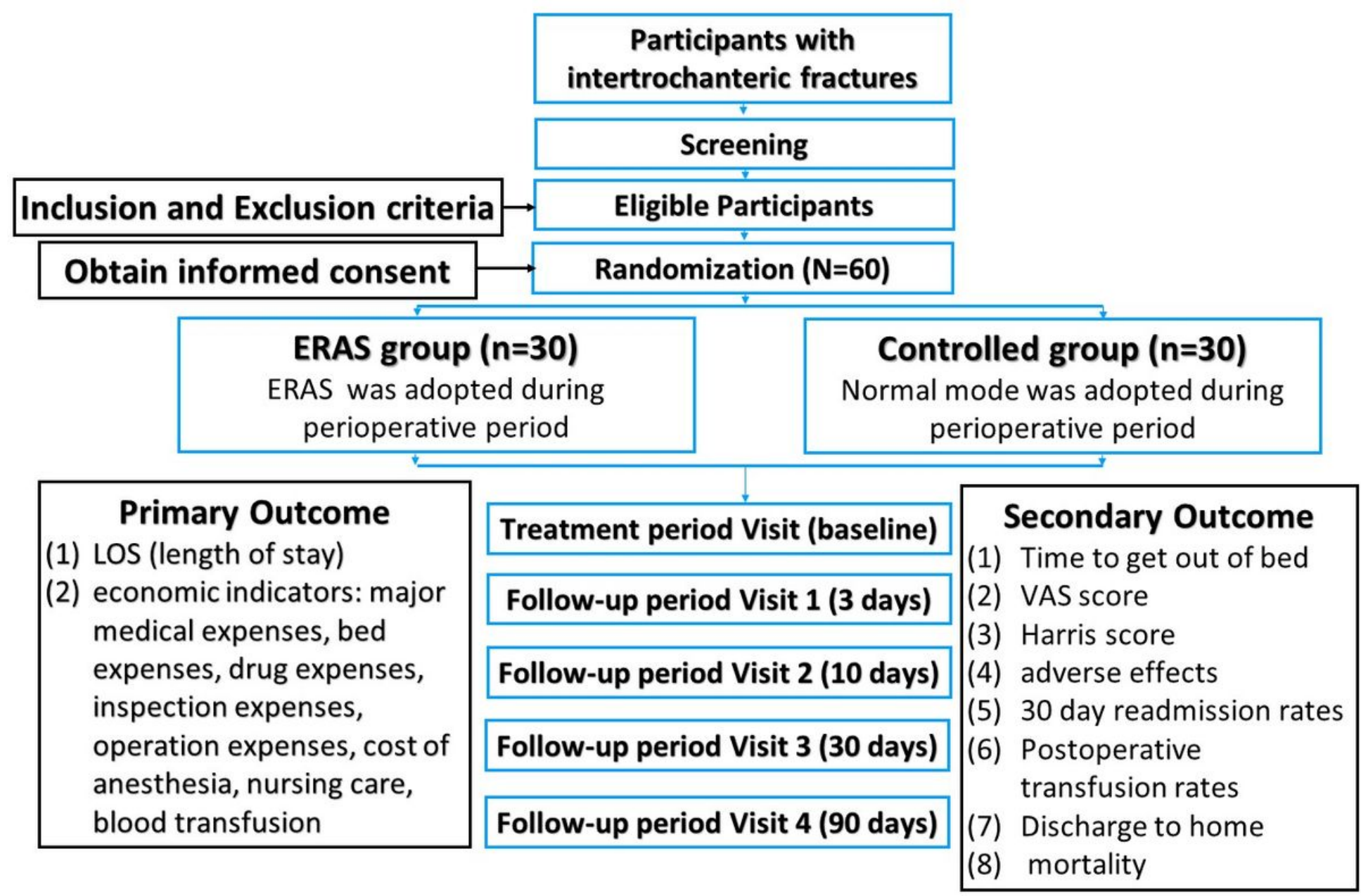

Figure 1

Participant processing and the schedule of evaluation. 